Cahiers de recherches médiévales

Journal of medieval studies

L'héritage de Chrétien de Troyes

\title{
Sens et conjointure armoriale dans le Lancelot propre
}

Donald Maddox

\section{(2) OpenEdition \\ 12 Journals}

Édition électronique

URL : https://journals.openedition.org/crm/2657

DOI : $10.4000 / \mathrm{crm} .2657$

ISSN : 1955-2424

Éditeur

Honoré Champion

\section{Édition imprimée}

Date de publication : 15 décembre 2007

Pagination : 87-100

ISSN : 1272-9752

\section{Référence électronique}

Donald Maddox, "Sens et conjointure armoriale dans le Lancelot propre ", Cahiers de recherches

médiévales [En ligne], 14 | 2007, mis en ligne le 15 décembre 2010, consulté le 15 décembre 2022.

URL : http://journals.openedition.org/crm/2657 ; DOI : https://doi.org/10.4000/crm.2657

Tous droits réservés 


\title{
酷M
}

\section{Sens et conjointure armoriale dans le Lancelot propre ${ }^{1}$}

\author{
à la mémoire d'Elspeth Kennedy
}

Dans son intégralité, le Lancelot propre est jalonné de détails évocateurs de l'héraldique médiévale à ses débuts ${ }^{2}$. Les nombreux écus portés par les chevaliers font partie d'un répertoire assez important de propriétés descriptives qui, servant tantôt à maintenir un certain "effet de réel " chevaleresque, tantôt à alimenter les puissants contre-courants du merveilleux, concourent à consacrer l'étrange parmi les registres stylistiques qui prédominent à travers le cycle du Lancelot-Graal. Quoique cet aspect du Lancelot propre attire l'attention des chercheurs depuis un certain temps - par exemple, C. Dover analyse les quatre écus magiques offerts au héros éponyme par la Dame du $\mathrm{Lac}^{3}, \mathrm{M}$. de Combarieu du Grès dresse une taxinomie des «couleurs de l'héraldique » et des "écus portés par les héros» du Lancelot-Graal ${ }^{4}$ il reste toutefois à démontrer que de tels éléments sont pris en charge selon un principe d'organisation narrative compréhensive et par là acquièrent une importance tout à fait exceptionnelle dans la première partie du Lancelot propre. Celle-ci correspond à la soi-disant version «non-cyclique $»^{5}$, qui raconte l'enfance du héros,

\footnotetext{
1 J'ai présenté une version abrégée de cet essai à Toulouse, lors du XIXe Congrès international de la Société Internationale Arthurienne, le 26 juillet 1999.

${ }^{2}$ Sur les origines de l'héraldique médiévale, voir M. Pastoureau, Traité d'héraldique, Paris, Picard, 2003, p. 20-36, et G. J. Brault, Early Blazon, Heraldic Terminology in the Twelfth and Thirteenth Centuries, with Special Reference to Arthurian Literature, Oxford, The Clarendon Press, 1972, p. 29-35.

${ }^{3}$ C. Dover, "The Split-Shield Motif in the Old French Prose Lancelot», The Arthurian Yearbook, New York, Garland, 1991, p. 43-61. En limitant l'enquête ainsi, l'auteur passe sous silence la quasi-totalité des écus portés par le héros éponyme.

${ }^{4} \mathrm{M}$. de Combarieu du Grès, D'aventure en aventure: 'semblances' et 'senefiances' dans le Lancelot en prose, Aix-en-Provence, CUERMA, 2000, p. 235-362, 275-94, et 360-62.

${ }^{5}$ Il s'agit du manuscrit BNF fr. 768 dont le dernier folio manque, lacune supléé par deux autres manuscrits, Rouen, Bibliothèque Municipale 1055 et Florence, Biblioteca MediceoLaurenziana, Laur. 89. Inf. 61. Sur la tradition manuscrite, voir E. M. Kennedy, éd., Lancelot do Lac: The Non-Cyclic Old French Prose Romance, 2 t., Oxford, The Clarendon Press, 1980 , t. 2, p. 29-31. En ce qui concerne le contenu, «[t]he non-cyclic romance contains an account of Lancelot's childhood and education and the knightly adventures through which he discovers his own name, establishes a reputation under several different designations (White Knight, Red Knight, Black Knight, etc.), saves Arthur's kingdom, and wins the love of Guinevere and a seat at the Round Table (a narrative common to both non-cyclic and cyclic versions). This is followed in the non-cyclic romance by a short version of Lancelot's journey with Galehot to Sorelois, during which Galehot has dreams foretelling his death, and of the False Guinevere episode in which Lancelot defends the Queen against the charges of an impostor; the romance is brought to a conclusion with the death of Galehot, arising from his separation from Lancelot». E. Kennedy, «The Re-Writing and Re-Reading of a Text: the
}

Cahiers de Recherches Médiévales, 14, 2007 
placé très tôt sous la tutelle de la Dame du Lac, jusqu'à l'apogée de sa carrière chevaleresque, marquée par la sanction collective suprême que lui décerne le roi Arthur: l'installation à la Table Ronde ${ }^{6}$. Mettant en relief d'une part la construction progressive de l'identité héroïque - rien moins que superlative - de Lancelot, d'autre part l'amour réciproque qu'il partage avec la reine, ${ }^{7}$ cette partie du Lancelot propre incorpore - on se propose de le démontrer ici- une «architecture héraldique» dont la pierre angulaire est le personnage éponyme lui-même. Car parmi le grand nombre d'écus diversement peints et d'armes distinctivement colorées qui en échelonnent le parcours narratif, ceux portés successivement par Lancelot en viennent à signifier en tant qu'ensemble. Dans ce qui s'ensuit, il sera donc question des fonctions et du sens de cette «conjointure armoriale» qui soustend l'organisation intégrale du Lancelot propre dans sa dimension non-cyclique.

\section{Les Écus de Lancelot}

Situons d'abord, par rapport au parcours narratif de la première partie du Lancelot propre, les blasonnements qui conviennent aux écus et aux armes affichés successivement par Lancelot.

1. d'argent plain $(48 \mathrm{~d}, 50 \mathrm{~b}, 60 \mathrm{~d}, 61 \mathrm{~d}, 65 \mathrm{c}, 65 \mathrm{~d}, 66 \mathrm{~d})^{8}$ :

Le jeune héros éponyme arrive à la cour du roi Arthur pour la première fois vêtu d'armes monochromes, don de Ninienne, la Dame du Lac, pour son adoubement'. Comme d'autres chevaliers nouveaux dans le Lancelot propre, il revêt

Evolution of the Prose Lancelot", The Changing Face of Arthurian Romance: Essays in Memory of Cedric E. Pickford, éd. A. Adams et al., Cambridge, England, D. S. Brewer, 1986, p. 3.

${ }^{6}$ Ceci a lieu à la suite de la guerre du royaume arthurien contre les Saxons et peu avant la mort de Galehaut. D'où, semble-t-il, un Lancelot propre « liminaire », antérieur à l'élaboration de la version "cyclique », qui comporte notamment la "préparation» à la Queste del Saint Graal et la relativisation de l'image de Lancelot, qui semblera désormais crépusculaire par rapport à celle, déjà exaltée, de son fils, Galaad. «In the cyclic version, the death of Galehot no longer forms the conclusion to the work, the Journey to Sorelois and the False Guinevere episodes are re-written to prepare the way for the Charrette story and a Galahad Quest for the Grail, and the interpretation of Galehot's dreams both presents the love of Lancelot and Guinevere for the first time in the romance as destructive and sinful, and predicts the coming of a knight, pure and chaste descendant of Lancelot and destined to surpass him ». Kennedy, «Re-Writing», p. 3. Voir aussi E. M. Kennedy, Lancelot and the Grail : a study of the Prose Lancelot, Oxford, The Clarendon Press, New York, Oxford University Press, 1986, Part II, «Lancelot without the Grail», p. 5-249.

${ }^{7}$ Kennedy, Lancelot and the Grail, p. 10-78.

${ }^{8}$ Pour donner une idée de la fréquence relative de chaque écu, les notations entre parenthèses renvoient soit au manuscrit BNF fr. 768 soit, précédé d'un J, au manuscrit British Library BL Add 10292. Il s'agit des folios comportant une évocation qui correspond au blason indiqué.

9 «Le port d'un écu monochrome par les jeunes chevaliers pendant l'année qui suit leur adoubement est en effet un thème cher à l'héraldique littéraire du XIIIe siècle». $M$. Pastoureau, «Les armoiries de Tristan dans la littérature et l'iconographie médiévales », 
d'abord des armes blanches, selon la coutume ${ }^{10}$. Or l'écu d'argent plain signifie pour autant le stade initial, "non-marqué ", de la carrière chevaleresque, table rase armoriale à être éventuellement «redorée », à plusieurs reprises, toujours en fonction des aventures ultérieures.

2. d'argent, à une bande vermeille (62d, 64a, 69b, 70d, 107d) :

Lors de sa première grande épreuve, à la Douloureuse Garde, «li Blans Chevaliers » abat cinq gardiens du château avant que Ninienne ne lui envoie trois écus magiques pour qu'il puisse lutter plus efficacement contre les enchantements exceptionnellement formidables qui pèsent sur ce lieu maléfique ${ }^{11}$. Chacun des trois écus étant au champ d'argent associé à son adoubement, le premier, à une bande vermeille, doublera sa force : «si tost com vos avroiz au col celui o il n'a que une seule bande, si avroiz recovree la proesce et la force d'un chevalier avec celi que vos avez» (Lp, p. 188, M, VII, p. 321). En effet, dès que l'écuyer le lui apporte, "lors sant sa force doblee, si est tant vistes et tant legiers que il ne se sant de cop ne de plaie que il ait. Et tantost laisse corre a els toz et fiert destre et senestre, et fait tels mervoilles que nus ne lo voit qui ne s'an esbaïsse » (Lp, p. 191, M, VII, p. 327).

3. d'argent, à deux bandes vermeilles $(62 \mathrm{~d}, 64 \mathrm{~b}, 72 \mathrm{a}, 107 \mathrm{~d})$ :

Vers tierce, toutefois, le héros commence à s'épuiser; or, la demoiselle de Ninienne lui apporte l'écu à deux bandes vermeilles, et il se trouve ranimé par «la proesce a deus chevaliers» (Lp, p. 188, M, VII, p. 321), ce qui lui permet d'avancer vers la porte principale du château avant d'être à nouveau ralenti par d'autres assaillants.

4. d'argent, à trois bandes vermeilles $(62 \mathrm{~d}, 64 \mathrm{c}, 72 \mathrm{~b}, \mathrm{~J} 50 \mathrm{~d}, \mathrm{~J} 51 \mathrm{e}, 107 \mathrm{~d})$ :

Enfin, l'écu à trois bandes vermeilles quadruple son pouvoir en lui procurant «la proesce a trois chevaliers» (Lp, p. 188, M, VII, p. 321) pour neutraliser une dernière vague d'assaillants et dégager la porte d'entrée. Maintenant à l'apogée de son pouvoir augmenté de forces surnaturelles, il subit l'aventure, hautement identitaire, du cimetière merveilleux où, en ouvrant la tombe qui lui est réservée, il apprend son nom et celui de son père et puis reçoit un accueil triomphal au palais seigneurial de la Douloureuse Garde (Lp, p. 191-95, M, VII, p. 326-33). Lorsque par la suite le roi Arthur et son entourage essaient d'y entrer, Lancelot, faisant fonction de portier malgré lui, se munit à nouveau des trois écus à tour de rôle, ce qui empêche la reine de le reconnaître (Lp, p. 205-17, M, VII, p. 349-69). Aussi ce trio armorial, le dotant d'abord d'auxiliaires merveilleux puis d' "anseignes" dont il se sert lui-même pour dissimuler son identité, démontre-t-il d'une part combien la

Gwéchall, Bulletin de la Société finistérienne d'histoire et d'archéologie, I, 1978, p. 15. Sur l'usage littéraire des écus monochromes, ou «plains », voir Brault, Early Blazon, p. 29-35.

${ }^{10}$ Cette coutume est évoquée plus tard lors de l'adoubement du jeune bachelier, Hélain : «li escu estoit toz blans comme nois, si com a cel tans estoit costume que chevaliers noviaus portoit escu d'un sol taint lo premier an que il estoit» (Lp, p. 395, M, VIII, p. 194). Les citations du Lancelot propre (= Lp) proviennent de Kennedy, éd., Lancelot do lac, tome premier. Le sigle « $\mathrm{M} »$ renvoie au passage analogue dans Lancelot, roman en prose $d u$ XIII ${ }^{e}$ siècle, éd. A. Micha, Paris-Genève, Droz, 1980 (t. VII) ou 1982 (t. VIII).

${ }^{11} \mathrm{Ce}$ chevalier nouveau doit, selon la messagère de Ninienne, éviter de faire confiance à sa seule «joventé » (Lp, p. 395, M, VII, p. 194). 
Dame du Lac tient à gérer le déroulement de sa qualification chevaleresque et identitaire, d'autre part chez Lancelot un esprit d'autonomie maligne et calculatrice dont il se servira souvent au cours de ses aventures ultérieures.

\section{5. de sinople, à une bande d'argent (J 51e, 73c) :}

S'ensuivent une longue série d'aventures marquées elles aussi par son souci d'anonymat. Paradoxalement, là où l'héraldique médiévale servait à révéler l'identité de leur détenteur, Lancelot s'en servait souvent pour cacher la sienne. D'où l'éclatement de ce personnage parmi toute une variété d'identités empruntées et totalement factices ${ }^{12}$. Quoique C. Dover suggère que Lancelot veut rester anonyme parce que «he has not yet reached a sufficiently glorious level of achievement to warrant disclosure or because disclosure would restrict his freedom of movement or endanger his love $»^{13}$, il y a pourtant une raison bien plus significative : Lancelot se considère chevalier exclusivement de la reine; or, il lui importe surtout que ce soit elle qui le reconnaisse, en pénétrant son identité par le biais de ses écus' ${ }^{14}$. Ainsi recouvre-t-il son écu d'une housse (Lp, p. 220, M, VII, p. 373), puis l'échange contre un écu de sinople à une bande d'argent (Lp, p. 224, M, VII, p. 379) ce qui, en inversant le teint du champ et celui de la bande, amoindrit l'espace consacré à l'argent par rapport au sinople, teint évocateur du sang ${ }^{15}$. D'où à ce stade une connotation symbolique : muni de cet écu, Lancelot doit tuer plusieurs chevaliers - qui sont des hommes valeureux, d'ailleurs - par respect du vœu qu'il avait solennellement juré au «chevalier enferré»(Lp, p. 160, M, VII, p. 278). L'arrivée de celui-ci à la cour avait coïncidé avec celle de Ninienne et le jeune héros: couché dans une litière, cet inconnu, le corps pénétré de deux tronçons de lance avec leurs fers et dans sa tête une épée, exige que celui qui le déferrera devra jurer «sor sainz qu'il me vanchera a son pooir de toz cels qui diront qu'il ameront plus celui qui ce me fist que moi» (Lp, p. 150, M, VII, p. 263). Seul ce jeune bachelier ose émettre un vœu tellement inconsidéré et, avant même de se faire attribuer l'épée consacrant son adoubement, il déferre ce chevalier et pour autant se transforme ultérieurement, et à plusieurs reprises, en instrument de vengeance de la part de «l'enferré» et son lignage, contre des parents de l'agresseur de celui-ci. Malgré l'ambiguïté de ce vœu insolite, les épisodes qui en découlent concourent à affirmer que Lancelot est bien «le meilleur chevalier du monde $»^{16}$.

\footnotetext{
${ }^{12}$ L'auteur souligne cet effet à plusieurs reprises, précisant, par exemple, que pendant la guerre contre les Saxons les déplacements rapides de Lancelot au champ de bataille créaient chez l'ennemi l'impression que plusieurs chevaliers, tous aux armes identiques, s'exerçaient simultanément (Lp, p. 561, M, VIII, p. 469).

${ }^{13}$ Dover, «The Split-Shield Motif», p. 46.

${ }^{14}$ Voir, p. ex., les propos qu'il tient à un autre chevalier: "Sire, de par qui merchierai je madame la roine . . . car je ne sai comment vos avés non?-Mon escu li devisés, fait il, car mon non ne poés vous savoir, et bien li dites que par li estes delivrés » (Lp, p. 223, M, VII, p. 377).

${ }^{15}$ Le terme sinople est d'abord synonyme de gules ou vermeille et n'acquiert que vers le troisième quart du 14e siècle le sens de vair (vert). Brault, Early Blazon, p. 275-76.

${ }^{16}$ Voir M.-L. Chênerie, «L'aventure du chevalier enferré, ses suites et le thème des géants dans le Lancelot», et F. Suard, "Lancelot et le chevalier enferré", dans Approches du Lancelot en prose, éd. J. Dufournet, Paris, Champion, 1984, p. 59-99 et 177-96. Voir aussi
} 
6. de sinople plain $(74 \mathrm{~b}, 74 \mathrm{~d}, 79 \mathrm{~b}, 82 \mathrm{a}, 89 \mathrm{a}, 89 \mathrm{~b}, 89 \mathrm{c}, 93 \mathrm{a}, 95 \mathrm{c}, 99 \mathrm{~d}, 100 \mathrm{~b}$, 108a, 108d, 110a, 113b) :

À la «première assemblee», tournoi co-parrainé par le roi Arthur, il l'emporte sur tous en affichant le rouge monochrome, de sinople plain. C'est le premier écu totalement indépendant de tout souvenir chromatique des «dons blancs» de sa marraine et par là de son état de chevalier-novice. Évoqué bien plus souvent que les autres écus portés par Lancelot, cet « escu vermoil » met au premier plan le profil d'un chevalier enfin mûr, rôdé, et il semble également receler une connotation symbolique du côté violent, et pour ainsi dire «sanguinaire», de la vocation chevaleresque. On ne s'étonne donc pas qu'il retrouve son incognito en recouvrant cet écu d'une housse (Lp, p. 246, M, VII, p. 412) avant de partir au secours de la reine, qu'il croit, à tort, être prisonnière à la Douloureuse Garde, ce qui lui permet, néanmoins, d'en lever les enchantements (Lp, p. 248-50, M, VII, p. 414$18)$.

\section{7. d'argent, à une bande de sable $(80 \mathrm{c}, 81 \mathrm{c})$ :}

À une seconde «assemblee», il se munit d'un écu d'argent, à une bande de sable (noir) et accomplit une première journée prodigieuse contre tous ses adversaires. Toutefois, cette «valse aux écus» menée par une pluralité de chevaliers factices s'avère soudain moins efficace : ayant appris d'une demoiselle, et devant Lancelot lui-même, l'identité du vainqueur des deux assemblées (Lp, p. 255, M, VII, p. 426), Gauvain en fait état à la cour arthurienne: «Qui fu, fait li rois, li chevaliers qui nos fist antrer en la Dolereuse Garde? - Ce fu, fait il, Lanceloz del Lac, li filz au roi Ban de Benoyc. Et ce fu cil qui vainquié l'asenblee de vos et del Roi d'Outre les Marches, qant il porta les armes vermoilles, et ceste dont nos venons ra il vaincue » (Lp, p. 256, M, VII, p. 428). Par conséquent, Lancelot ne participe plus à la seconde assemblée, «car il cremoit estre delaiez» (Lp, p. 256, M, VII, p. 427). Comme on voit à plusieurs reprises, seule l'intéresse le moment où la reine le reconnaîtra.

8. de sable plain : (96b, 99d, 100b, 105a, 107b, 108d, 110a) :

Prisonnier ensuite de la Dame de Malohaut, il profite des deux congés qu'elle lui accorde pour briller lors de la guerre entre le roi Arthur et Galehaut, qui nourrissait un grave différend avec le royaume arthurien. Lors du premier affrontement il est muni à nouveau de l'écu de sinople plain; au second il porte un écu de sable plain. Ce passage abrupt du rouge au noir monochrome, teint souvent associé au mal, n'occasionne pourtant pas chez le public qui assiste aux affrontements chevaleresques la moindre réticence à l'égard de ce «Noir Chevalier ». Au contraire, on l'estime mieux que son prédécesseur de sinople plain : «Et dient tuit cil qui ses proesces ont veües que neianz fu de celui d'antan as armes vermoilles envers cestui»(Lp, p. 315, M, VIII,p. 65). Au lecteur, le seul averti, d'apprécier ce détail à sa juste valeur : «Plus ça change . . .»!

9. d'or, à un lioncel de sinople, les armes de Galehaut (103a, 107b, 163a) :

Or grâce justement à l'amitié naissante entre ce "Chevalier Noir» et Galehaut, celui-ci décide de conclure la paix avec Arthur, et Lancelot, toujours anonyme, marque le début de leur amitié en revêtant les armes de Galehaut - d'or, à

L. Jefferson, Oaths, Vows and Promises in the First Part of the French Prose Lancelot Romance, Bern, Peter Lang, 1993, p. 103-32. 
un lionceau de sinople. Cette profonde amitié ainsi liée avec Galehaut fournit un médiateur - tiers indispensable - pour arranger le célèbre entretien de Lancelot et la reine, «colloque» on ne peut plus sentimental, où il est souvent question de la diversité des armes naguère en vue, et par là du moment où la reine reconnaît enfin ce «chevalier à maintes écus» et lui décerne sa récompense amoureuse. Nous y reviendrons.

10. de sable, à une bande d'argent (160c, 164b, 164d, 171c) :

À la suite de cette belle conjoncture affective, Lancelot change moins souvent d'armes. Lors de l'invasion des Saxons, la reine préscrit qu'il porte un écu de sable, à une bande d'argent, comme si elle y apposait une sorte de «brisure», symbolisant d'une part leur alliance affective, d'autre part les prodiges du «Noir Chevalier» qui avait sauvé le royaume. Par ailleurs, elle lui envoie des «druëries »", des cadeaux qui signifient sa dévotion amoureuse: une broche pour fermer son encolure, un anneau, un peigne précieux, sa ceinture, et surtout une "connoissance», un petit pennon à une pointe en soie vermeille (Lp, p. 525, M, VIII, p. 408), pour qu'il l'affiche à son heaume en guise de "reconnaissance», ce qui lui permet de le reconnaître lors de l'assemblée contre les Saxons. Selon le narrateur, ce fut «la premiere connoissance que onques fust portee au tans le roi Artu sor hiaume» (Lp, p. 542, M, VIII, p. 437), notation pseudo-historique qui suggère une conscience historique de l'héraldique, aussi bien qu'un certain ludisme esthétique.

11. parti, au $1:$ de ... au chevalier ... ; au 2 : de ... à la dame ... (125c, 127a, 132a, 166b, 168b, 168c, 168d, 169a, 169b, 171c) :

Le dernier écu dans cette série est le plus explicitement emblématique, et le plus étrange. Divisé verticalement - donc parti - mais pour une raison d'ordre nonhéraldique, l'écu est littéralement fendu: «il est toz fanduz des lo pié jusque an la panne amont ... . et sont les deus moitiez si loim l'une de l'autre que l'am puet antredeus fichier sa main sanz tochier as deux moitiez » (Lp, p. 402, M, VIII, p. 206). Écu figural, en plus : il affiche un chevalier et une dame «affrontés ${ }^{18}$ : "En l'une des parties de l'escu avoit un chevalier si richement armé com cil lo sot miauz faire qui lo fist, fors la teste. Et an l'autre moitié estoit portraite une si belle dame com an la pot plus belle portraire, si estoit par an haut si pres a pres que li uns tenoit les braz au col a l'autre et s'antrebaissasent, se ne fust la fandeüre de l'escu. Mais par desoz estoient si loign a loign com plus pueent» (Lp, p. 402-3, M, VIII, p. 206). D'où le blason, d'ailleurs inachevé, faute des teints, parti, au $1:$... au chevalier ...; au 2 : à la dame ... affrontés. Décerné cette fois non au héros mais à la reine, l'écu féndu est, encore une fois, un don de Ninienne, désormais médiatrice de cette liaison. Ignorant d'abord la source de ce fort curieux objet, la reine en constate d'emblée son état d'imperfection : «cist escuz est mout cortois, se il ne fust si fanduz», mais non sans

\footnotetext{
${ }^{17}$ Voir Roger Dubuis, «La notion de druërie dans les Lais de Marie de France », Le Moyen Age, 98, 1992, p. 391-413.

${ }^{18}$ Terme qui, en français, désigne d'habitude deux animaux face-à-face. Voir M. Popoff, E. de Boos, M. Chatenet, et C. Davy, Les armoiries, lecture et identification [Inventaire général des Monuments et des Richesses artistiques de la France], Poitiers, Centre d'Études Supérieures de Civilisation Médiévale, s.d. Voir aussi Brault, Early Blazon, p. 229, et Pastoureau, Traité, p. 358 .
} 
l'admirer aussi - «il pert estre toz fres»(Lp, p. 403, M, VIII, p. 207). Ignorant d'abord la source, elle en apprend la «senefiance» de la demoiselle qui le lui livre, sous forme de récit étiologique: «Dame, cil est uns chevaliers, li miaudres qui orandroit soit, [qui pria une dame d'amors, la plus vaillant qui orandroit soit] au mien cuidier. Tant fist li chevaliers, que par amor, que par ovre, li dona sa dame s'amor, mais plus n'i a ancor aü que de baisier et d'acoler, si comme vos veez an cest escu» (Lp, p.403, M, VIII, p. 207). Analepse évocatrice de ce qu'a vécu le couple jusqu'alors, ce récit est également l'ekphrasis d'une œuvre d'art éminemment digne de contemplation, pourvoyeuse d'esthésies, du moins la reine la perçoit-elle ainsi : «Et l'escu que la pucele avoit aporté fist pandre an sa chambre, si que ele lo veoit totjorz, car mout se delitoit an lui voir. Ne onques puis n'ala nul leu que il ne fust aportez devant li et panduz an sa chambre . . « (Lp, p. 407, M, VIII, p. 214). La demoiselle lui explique en outre que c'est un objet à valeur proleptique, comportant une échéance : «Et qant il avanra que l'amors sera anterine, si sachiez que cist escuz que voz veez si desjoint se rajoindra et tanront ansenble ces deus parties» (Lp, p. 403, M, VIII, p. 207). Le sens principal en est limpide : quoique ce chevalier et sa dame soient prêts à s'étreindre, la brèche qui les sépare les en empêche, du moins provisoirement; leur amour charnel n'est que virtuel. Cet écu est donc une configuration rétro-prospective de l'histoire affective de Lancelot et la reine, depuis les stades progressifs de leur innamoramento jusqu'à la consommation physique de leur passion ${ }^{19}$. En plus, dans la mesure où la brèche qui le scinde en deux va se souder lorsque l'union charnelle sera enfin consommée - «anterine»c'est aussi une merveille pour ainsi dire «kinésio-mimétique » de ce moment exquis. Mais l'écu est carrément merveilleux à plus d'un titre : il guérira Lancelot de sa folie et, lors de la guerre contre les Saxons, l'aidera à semer l'épouvante parmi l'ennemi ${ }^{20}$. Symbole à la fois de l'union amoureuse à son stade de perfection et de l'amour en tant que force motrice de la prouesse maximale, consacrée au salut du royaume lors de son plus grand péril, l'invasion saxonne, l'écu dans son état pour ainsi dire «défendu» en vient à emblématiser doublement ce que fit ce meilleur chevalier du monde, «que par amor, que par ovre».

Or, ayant dans un premier temps revu le parcours narratif du Lancelot noncyclique selon la perspective armoriale, comment en évaluer la «conjointure» et le sens? Disons d'emblée que cette longue suite d'écus diversement blasonnés ne semble pas renvoyer à un milieu sociopolitique précis ${ }^{21}$. Plutôt s'agit-il de ce que M. Pastoureau a qualifié d' " héraldique imaginaire » qui, à partir de la fin du XII ${ }^{\mathrm{e}}$ siècle,

\footnotetext{
${ }^{19}$ Vient à l'esprit la tombe destinée à Lancelot et qu'il ouvre lui-même, dont le contenu écrit, à la fois analeptique et proleptique, renvoie à sa naissance et à sa mort et pour autant devient un «chronotope », lieu emblématique de la temporalité intégrale du Lancelot-Graal.

${ }^{20}$ Voir l'analyse de C. Dover dans « The Split-Shield Motif», p. 48-57.

${ }^{21}$ On sait qu'à l'époque où paraît le roman, les blasons littéraires peuvent parfois être "véritables", en reprenant les armoiries appartenant à un individu ou à une lignée. Par exemple, à propos du Bel Inconnu, voir Brault, Early Blazon, p. 22-23, et A. Guerreau, «Renaud de Bâgé : Le Bel Inconnu, structure symbolique et signification sociale », Romania, 103, 1982, p. 28-34.
} 
se manifeste notamment dans la littérature arthurienne ${ }^{22}$. Selon Pastoureau, «chez les auteurs des deux générations qui suivent celle de Chrétien de Troyes . . . il s'agit presque toujours d'armoiries fictives, attribuées à des héros de roman ou à des créatures mythologiques ... [c'est] la littérature arthurienne, et plus particulièrement les versions en prose du début $\mathrm{du} \mathrm{XIII}^{\mathrm{e}}$ siècle, qui en ce domaine est la plus créatrice $»^{23}$.

Ainsi en est-il des armoiries conçues uniquement à l'usage de Lancelot, mais par qui ? Or, à la différence du «bricolage armorial » éphémère de Lancelot lors de ses tentatives de rester anonyme, dans la société féodale la conceptualisation des armoiries vraies s'effectuait typiquement selon les souhaits de leur détenteur, individuel ou collectif, et pour une durée en principe illimitée. Par contre, notre auteur délègue une part considérable de responsabilité de «l'invention» des armoiries de Lancelot à des personnages féminins. À elles s'attribue souvent, pour s'occuper de la «gestion» de cette héraldique imaginaire, la fonction de destinateur armorial. Donatrice des armes blanches, puis, par l'intermédiaire de sa demoiselle, des trois écus magiques, Ninienne garantit d'abord la réussite de Lancelot lors de sa conquête de la Douloureuse Garde. Si par la suite celui-ci jouit d'une plus grande liberté quant au choix de ses armes, la Dame de Malohaut le dote d'une panoplie rouge, puis des armes noires. Plus tard, la reine lui envoie son pennon, et lui propose, en souvenir de l'écu de sable plain avec lequel il avait brillé lors de la seconde assemblée contre Galehaut, le blason de sable, à une bande d'argent. Ensuite, c'est à nouveau la Dame du Lac qui remet les forces du merveilleux à la disposition de Lancelot, cette fois par le biais de l'écu fendu qu'elle offre d'abord à la reine, ce qui démontre à nouveau combien la carrière du «meilleur chevalier du monde » est redevable à l'ordre féerique qui intervient par moments pour la façonner et pour l'entériner. Pour cet aspect «armorial» de la première partie du Lancelot propre, comme pour bien d'autres aspects, d'ailleurs, l'auteur semble s'inspirer du Bel Inconnu ${ }^{24}$, dans la mesure où le héros bénéficie à plusieurs reprises d'un domaine de valeurs et d'adjuvants féminins, univers d'abord sous la tutelle surnaturelle et carrément maternelle de Ninienne, qui ne manque pas par la suite de surveiller et d'aider le héros au seuil de sa pleine maturité, parfois à l'insu de celuici, lors de sa transition vers l'établissement d'une relation amoureuse réciproque.

En ce qui concerne l'ensemble des composants de cette héraldique en quelque sorte "gynocentrique», il reste assez fruste en fait, la plupart des écus se

\footnotetext{
${ }^{22} \mathrm{M}$. Pastoureau, «Introduction à l'héraldique imaginaire », Revue française d'héraldique et de sigillographie, 48, 1978, p. 29-45. Voir Brault, Early Blazon, p. 18-54, Pastoureau, "Remarques sur les armoiries de Gauvain», Marche Romane, 30, 1980, p. 229-36 et, du même auteur, «Héraldique arthurienne et civilisation médiévale : notes sur les armoiries de Bohort et de Palamède ", Revue française d'héraldique et de sigillographie, 50, 1980, p. 2941.

${ }^{23}$ M. Pastoureau, Figures et couleurs : Étude sur la symbolique et la sensibilité médiévales, Paris, Le Léopard d'Or, 1986, p. 92, 98.

${ }^{24}$ Voir D. Maddox, "Les Armoiries de l'Inconnu», in Miscellanea Mediaevalia: Mélanges offerts à Philippe Ménard, éd. J.-C. Faucon, et al., Geneva: Slatkine, 1998, p. 933-42 et, en ce qui concerne le thème de l'identité dans les deux romans, E. Kennedy, Lancelot and the Grail, p. 10-48.
} 
caractérisant par la forte redondance de quelques éléments de base : la bande; un seul métal - l'argent; seulement deux teints - le sinople et le sable. Cependant, la variété d'emblèmes conçus autour de cette matière première minimale et l'habileté de leur agencement suggèrent qu'un vrai esprit de système est à l'ouvrage. Car entre l'écu initial et l'avant-dernier, la série traverse un chromatisme progressif qui passe graduellement du blanc au rouge, puis au noir, mais le plus souvent par des stades intermédiaires réalisés à l'aide des bandes. Cette métamorphose chromatique traduirait-elle ainsi quelque cohérence allégorique, ou bien quelque valorisation axiologique pareille, par exemple, au chromatisme des teints successifs du bois extrait de l'Arbre de la vie dans La Queste del saint Graal's ? Cela semble peu vraisemblable, en fait, surtout en ce qui concerne la progression blanc $>$ rouge $>$ noir, qui ne traduit aucun développement moral négatif tel, par exemple, que innocence $>$ violence sanguinaire $>$ déchéance. Tandis que Lancelot accomplit des exploits bénéfiques lorsqu'il porte des armes rouges, il est par la suite vêtu d'armes noires lors de ses prodiges effectués au service de la cour arthurienne, ceux-ci étant encore plus éblouissants que ceux qu'il avait réalisés en rouge à la Douloureuse Garde $^{26}$. Par contre, ces décalages entre un horizon d'attente chromatique somme toute assez banal et les vérités circonstancielles qu'il sert à camoufler cadrent parfaitement bien avec le projet de dissimuler un héros superlatif sous les apparences d'un chevalier quelconque ou même maléfique, ce qui ne manque pas de multiplier les « effets d'étrange» que cultive à dessein l'auteur.

Effectivement, ici comme ailleurs, on ressent à plusieurs reprises une nette tendance à attribuer aux armoiries un sens explicite, qu'il s'agisse de l'argent plain du nouveau chevalier ou de l'écu fendu représentant des amants virtuels, et ceci s'étend même par endroits aux personnages secondaires ${ }^{27}$. Bref, on est souvent amené à se poser la question du sens de cette héraldique «imaginaire » qui parcourt la quasi-totalité du Lancelot non-cyclique.

Or, en ce qui concerne les rapports entre sens et structuration intégrale, on s'aperçoit que ce segment du Lancelot propre incorpore le paradigme conventionnel du conte folklorique en tant que récit à trois épreuves. Aussi cette série d'écus se situe-t-elle entre l'épreuve qualifiante initiale, l'adoubement du héros - différé, bien sûr, pour que la reine puisse expédier l'épée nécessaire à son achèvement - et l'épreuve glorifiante, sanction positive de la reconnaissance de Lancelot que lui

\footnotetext{
${ }^{25}$ Voir M. de Combarieu du Grès, D'aventure en aventure, p. 339, 346-49.

${ }^{26}$ Dans le roman médiéval, le vermeil et le sable ne connotent pas forcément l'exercice d'une prouesse exceptionnelle. Selon Pastoureau, «le chevalier aux armes noires est un étranger ou quelqu'un qui cherche à cacher son identité ; le chevalier aux armes vermeilles est en général animé de mauvaises intentions ». "Remarques sur les armoiries de Gauvain», p. 230. Par contre, le rouge infléchit les écus de plusieurs chevaliers dans l'entourage de Lancelot, parmi eux Gauvain, d'or, à un lion de sinople (Lp, p. 408, M, VIII, p. 215), Girflet, de sinople, à une fasce d'or (Lp, p. 487, cf. M, VIII, p. 346), Galehaut, d'or, à un lioncel de sinople (Lp, p. 535, M, VIII, p. 425), Guinier, d'argent, à une fasce de sinople (Lp, p. 542, M, VIII, p. 436), et Yder, d'argent, semé de roues vermeilles (Lp, p. 550, M, VIII, p. 449).

${ }^{27}$ Voir, par exemple, l'étiologie de l'image du lion vermeil (Lp, p. 358, M, VIII, p. 132), et les gouttes d'argent figurant sur un champ noir qui signifient des larmes de tristesse (Lp, p. 375, M, VIII, p. 160-1).
} 
décernent à la fois la reine et le roi Arthur après sa réussite de l'épreuve décisive ou principale, lors de ses prestations chevaleresques pendant la campagne contre les Saxons. L'échelonnement des écus auxquels le héros a recours parcourt ce que le narrateur qualifie de «conte Lancelot» (Lp, p. 571, M, VIII, p. 488), segment ayant bien les dimensions d'un récit cohérent - semblable à ceux qui sous-tendent de nombreux romans courtois - qui traite de sa biographie chevaleresque entre son adoubement et son installation à la Table Ronde ${ }^{28}$.

Or dans ce "conte Lancelot» l'emploi assez systématique d'éléments héraldiques est justement une des caractéristiques les plus saillantes qui le distingue très nettement de la suite du roman. Quoique $M$. de Combarieu du Grès n'évoque pas la version «non-cyclique» du Lancelot propre, son relevé exhaustif des «couleurs de l'héraldique» dans l'intégralité du roman démontre d'une part la grande richesse des couleurs du blason dans les tomes VII et VIII de l'édition de Micha, ce qui correspond précisément à la version «non-cyclique», d'autre part qu'après le tome VIII, «les références aux couleurs du blason dans leur variété et leurs contrastes pittoresques, diminuent $»^{29}$, «ce qui se dessine progressivement, depuis le début du cycle, c'est une diminution des occurrences de couleur $»^{30}$. Le chromatisme de cet «esprit héraldique » qui prédomine dans le «conte Lancelot» et diminue par la suite semble donc renforcer l'hypothèse d'une version «liminaire » du Lancelot propre, antérieure à l'élaboration de la version «cyclique».

En outre, dans ce «conte Lancelot» le parcours héroïque se divise en deux grands volets, l'un portant plus directement sur sa vie privée, mûe par son obsession avec la reine, l'autre amalgamant la suite de cette intrigue passionnelle et la consolidation de son statut collectif, arthurien. Quant au premier volet, le jeu des écus se déroule entre son initiation à la vie chevalersque et sa rencontre avec la reine arrangée par Galehaut. Lors donc de ce premier entretien entre lui et sa bien-aimée (Lp, p. 339-46, M, VIII, p. 103-15), il s'agit d'une sorte d'interrogatoire, occasionnant en enfilade un résumé à deux qui reconstitue progressivement la première série des aventures de Lancelot, démarche qui permet à la reine d'extraire, au fur et à mesure, des renseignements concernant l'identité de son interlocuteur, aboutissant à la reconnaissance capitale où, toutes les identités signifiées par ces écus étant ramenées finalement à un seul et même chevalier, elle s'exclame : «Ha ! fait ele, donc sai ge bien qui vos iestes. Vos avez non Lanceloz do Lac» (Lp, p. 344, M, VIII, p. 109).

\footnotetext{
${ }^{28}$ Effectivement, l'évocation de l'installation de Lancelot, Galehaut et Hector à la Table Ronde coïncide avec la mention du «conte Lancelot» qui, selon le narrateur, est une «branche de Greal» (Lp, p. 571). Cf. Kennedy, «Re-Writing», p. 8: «the account of Lancelot's childhood and first adventures as a knight, that is up to his acceptance at Arthur's court under his own name and his installation at the Round Table, becomes, without changing the words of the text, one branch in a Lancelot-Grail cycle». Voir aussi Kennedy, Lancelot and the Grail, p. 152.

${ }^{29}$ M. de Combarieu du Grès, D'aventure en aventure, p. 292, et la liste des «séquences colorées » et des «pages 'riches' en notations de couleur» dans le Lancelot propre, La Queste del saint Graal, et la Mort Artu, p. 338-39.

${ }^{30}$ Ibid, p. 344.
} 
Dans ce passage comme dans bien d'autres, l'évocation de certains écus renvoie à des épisodes antécédents ou en occasionne le résumé, et ces analepses servent à rappeler au lecteur ou à l'auditeur ce qui s'est passé à tel ou tel endroit. Ainsi le système armorial du Lancelot propre non-cyclique rejoint-il d'autres dispositifs mnémoniques échelonnés à travers le roman ${ }^{31}$, d'où une espèce d' «art de mémoire », savamment aménagé par l'architecte du roman non-cyclique.

En outre, cette longue analepse diégétique par laquelle la reine et Lancelot reconstruisent ensemble, à l'aide de force repères armoriaux, les premières aventures de celui-ci offre un bel exemple du schéma, très répandu dans la littérature médiévale, que j'ai baptisé ailleurs la rencontre spéculaire. Il s'agit du moment où un personnage découvre soit un aspect de sa propre identité, soit des détails de son passé ou de son destin, toujours par moyen d'un interlocuteur qui lui fournit ces précisions tout à fait capitales ${ }^{32}$. D'une part, est spéculaire cette rencontre du fait que Lancelot redécouvre sa propre histoire comme si c'était dans un miroir, car il en entend la valorisation toute particulière dans le discours de la reine. C'est d'autre part une conjoncture doublement spéculaire puisque, de son côté aussi, elle commence à repenser certains moments de son propre passé qui se trouvent soudain auréolés bien autrement. Car avant ce premier intermède d'intimité amoureuse, elle avait perçu un décalage temporel entre les divers phénomènes armoriaux auxquels elle avait assistés et sa compréhension de la relation profonde entre eux. Cet entretien l'aide donc à réduire simultanément trois écarts : entre le présent et le passé ; entre Lancelot et tous ses déguisements; et, surtout, est réduit l'écart psychologique entre les deux personnages principaux. En plus, la reine comprend enfin combien son mot de congé, «A Dieu, fait ele, biax douz amis » (Lp, p. 165, 345, M, VII, p. 285, VIII, p. 111), qu'elle avait entendu dans un sens tout à fait anodin, avait profondément bouleversé la conscience de son interlocuteur extasié, dorénavant sous l'emprise d'une adoration inépuisable. Bref, cet entretien qui clôt le premier volet du «conte Lancelot» comble enfin l'écart cognitif entre la reine et son chevalier.

Le second volet débute par un nouveau départ du héros, cette fois pour Sorelois en compagnie de son ami Galehaut, ce qui déclenche au sein de la cour une crise, du fait que la quête pour le Chevalier Vermeil reste inachevée. La démultiplication des quêtes qui en découlent introduit un jeu armorial secondaire en quelque sorte, constitué par les écus appartenant aux chevaliers à la recherche de Lancelot. Cet enchevêtrement des parcours chevaleresques converge à nouveau vers la cour, pour que, lors de la guerre contre les Saxons, il y ait une mise en relief, spectaculaire et sans précédent, des prouesses de Lancelot, muni de l'écu que la reine lui a prescrit : de sable, à une bande d'argent. Puis, vers la fin de ce volet, au terme de la guerre, a lieu la seconde grande reconnaissance. À la différence de la première, clandestine, celle-ci est publique: avant l'installation de Lancelot à la Table Ronde (Lp, p. 571, M, VIII, p. 488), la reine, comme si elle ne le connaissait

\footnotetext{
${ }^{31}$ Voir par exemple D. Maddox, «A tombeau ouvert : Memory and Mortuary Monuments in the Prose Lancelot», in "Por le soie amisté »: Studies in Medieval Literature in Honor of Norris J. Lacy, ed. K. Busby and C. Jones. Amsterdam and Atlanta : Rodopi, 2000, p. 323-38. 32 Donald Maddox, Fictions of Identity in Medieval France, Cambridge, Cambridge University Press, 2000.
} 
pas encore, s'empare de son chevalier et l'embrasse devant toute la cour : «Et ele laisse toz les autres, si giete les braz a Lancelot au col, si lo baise voiant toz cels qui laianz estoient, por ce que toz les an voloit decevoir et que nuns n'i pansast ce qu'i est» (Lp, p. 569, M, VIII, p. 484), et devant tous elle lui avoue son amour -

«Sire chevaliers, ge ne sai qui vos iestes, ce poise moi ; ne ge ne vos sai que offrir por l'annor mon seignor avant et por la moie aprés, que vos avez hui maintenue. Mais por lui avant et por moi aprés vos otroi ge moi et m'amor, si comme leiaus dame doit doner a leial chevalier ». (Lp, p. 569, M, VIII, p. 484)

- puis feint son étonnement le plus profond lorsque le roi lui explique triomphalement que ce chevalier inconnu qu'elle comble de tant de louanges n'est autre que Lancelot du Lac. Son habileté, évocatrice ici de celle de l'Iseut de Béroul, lui permet de participer à une sanction bivalente de Lancelot, publique dans la mesure où elle seconde la sanction collective que lui décerne le roi Arthur, mais non sans réaffirmer celle, privée, qu'elle lui avait décernée en secret lorsqu'il lui avait avoué son amour. À deux reprises, donc, la conjointure armoriale sert à conditionner un moment crucial d'anagnorisis, en tant que reconnaissance principale qui survient vers la fin de chacun des deux volets.

Ainsi l'architecture compréhensive de ce «conte Lancelot» ressemble-t-elle pour autant à de nombreux romans arthuriens en vers dont le parcours intégral du héros se scinde en deux macro-segments, liés entre eux par une transition entre sa qualification individuelle et affective dans un premier temps et une suite représentant la croissance de son profil par rapport à une collectivité. Cette bipartition $^{33}$ est d'autant mieux mise en relief par les armoiries attribuées successivement à Lancelot et qui lui servent d'indices identitaires ${ }^{34}$. Ce qui par ailleurs fait tout l'intérêt de cette visée identitaire, on vient de le voir, c'est la tension que l'auteur maintient si habilement entre l'identité du héros et la dissimulation de celle-ci, toujours à l'aide du jeu des blasons. Soulignons en outre la solidarité thématique et figurale tout à fait remarquable entre les deux volets. Tout en ranimant le passé pour l'approfondir, la fin du premier volet qui comble l'écart subjectif entre Lancelot et la reine préfigure le point culminant du second, lorsque le dernier écu se soude, merveille qui fête chez le couple la réduction absolue entre eux de l'écart physique.

Voilà donc un «quasi-roman» en quelque sorte, situé pour ainsi dire «en abyme ", diptyque narratif élégamment serti à l'intérieur du grand Lancelot en prose, et dont la série armoriale qui le parcourt aboutit, au terme de chacun des deux panneaux du diptyque, au moment exquis où un écart chez le couple, l'un cognitif, l'autre charnel, est fermé. Ainsi l'intrigue amoureuse du Lancelot non-cyclique peint-il le passage d'un carrefour affectif à son analogue physique, la première rencontre à la fois armoriale et amoureuse en anticipant la seconde, celle qui marque

\footnotetext{
${ }^{33}$ Voir D. Maddox, «Medieval Textualities and Intergeneric Form», L'Esprit Créateur, 33, 1993, p. 40-50.

${ }^{34}$ E. Kennedy a bien évoqué les divers écus que porte Lancelot au long de son périple chevaleresque par rapport à ce qu'elle appelle «the identity theme», par moyen duquel l'auteur construit le personnage éponyme. Lancelot and the Grail, p. 10-48.
} 
enfin la perfection de ce couple ${ }^{35}$. Ainsi, d'un moment spéculaire à l'autre, le récit passe-t-il de la conjoncture in verbis à sa réplique figurale, éternisée par une merveille d'artisanat féerique. La bipartition du Lancelot propre non-cyclique s'accuse donc par cette analogie profonde entre la conjoncture amoureuse et la conjointure armoriale qui s'achève à ces deux moments-clé.

Il nous reste toutefois à souligner le fait que si la symétrie quasialgorithmique qui organise cette double «arthurianisation»- à la fois chevaleresque et amoureuse - de Lancelot, établissant ainsi sa relation significative et avec la reine et avec le roi, fait penser tellement à la bipartition de certains romans en vers, elle ne garantit pas pour autant la clôture du Lancelot non-cyclique, car celui-ci recèle des virtualités à être exploitées ultérieurement et dont le développement alimentera la puissante expansion cyclique du Lancelot propre. Car la «perfection» de ce «conte Lancelot $»^{36}$ que l'on vient de dégager ne concerne qu'un seul niveau de cohérence, celui de l'intrigue amoureuse, signifiée de façon emblématique, tandis qu'à d'autres niveaux prolifèrent les éléments de l'imperfection indispensable à l'ouverture de ce segment vers la suite du Lancelot propre dans sa dimension cyclique, ${ }^{37}$ notamment ceux qui concernent l'irrésolution de la carrière de Lancelot en tant que chevalier de la Table Ronde, virtualité à peine amorcée à ce stade.

Malgré donc l'emprise qu'auront exercée les possibilités latentes et fécondes dans ce texte pour la plupart «liminaire» sur l'esprit du (ou des) continuateur(s) du Lancelot propre dans son intégralité, nous sommes en droit d'apprécier en lui-même, tel un "Combray» proustien, la beauté et l'intégrité artistique de ce long segment initial de la biographie de Lancelot, celui qui dépeint la première floraison de sa

\footnotetext{
${ }^{35}$ L'écu enfin intégral représente l'archétype conceptuel du couple dans son état de perfection, « anterine ». Sur la notion de perfection, voir D. Kelly, The Art of Medieval French Romance, Madison, the University of Wisconsin Press, 1992, «Realization of the Romance : Parfaire », p. 134-45.

${ }^{36}$ Le «conte Lancelot» est le modèle conceptuel à la base du Lancelot propre dans son état non-cyclique. Sur la perfection et le roman inachevé, voir D. Kelly, The Art of Medieval French Romance, p. 136-43.

37 Voir P. V. Rockwell, "Je ne suiz mie soffisanz: Insufficiency and Cyclicity in the Lancelot-Grail Cycle», in Transtextualities, of Cycles and Cyclicity in Medieval French Literature, ed. S. Sturm-Maddox et D. Maddox, Binghamton, New York, Medieval \& Renaissance Texts and Studies, 1996, p. 71-91.
} 
carrière de chevalier et qui, quoiqu'il se déroule par un principe métonymique susceptible de lui assurer un avenir de longue haleine, est savamment rythmé par un puissant principe métaphorique, fondé sur une très belle conjugaison d'armes et d'amour.

Donald Maddox University of Massachusetts, Amherst 